\title{
ON THE SEISMIC SIGNATURE OF THE HEII IONIZATION ZONE IN STELLAR ENVELOPES
}

\author{
MÁRIO J.P.F.G. MONTEIRO \\ DMA-FCUP and CAUP, Universidade do Porto, Portugal
}

AND

\author{
MICHAEL J. THOMPSON \\ Astronomy Unit, QMW, University of London, England
}

Sharp variations of the structure of the star create a characteristic signal in its frequencies of oscillation (e.g. [3]). The zone of the second ionization of helium is such a localized feature of the structure whose properties depend mainly on the abundance of helium and the equation of state. Considering that such a signal should easily be detectable provided the frequencies are measured to rather better than $1 \mu \mathrm{Hz}$ accuracy (the COROT project should measure oscillation frequencies with an accuracy of $0.1 \mu \mathrm{Hz}$ ), we present here a tool to study this aspect of stellar structure.

The second ionization zone of helium causes a distinct 'bump' $\delta \Gamma_{1}$ in the adiabatic exponent $\Gamma_{1}$. This gives rise to a perturbation $\delta \omega$ of the frequencies (relative to a fictitious star without such a bump),

$$
\delta \omega \sim-\frac{3}{4 \tau_{\mathrm{t}}}\left(\frac{\delta \Gamma_{1}}{\Gamma_{1}}\right)_{\bar{\tau}_{\mathrm{d}}} \frac{\sin ^{2}(\omega \beta)}{(\omega \beta)} \cos \left[2\left(\omega \bar{\tau}_{\mathrm{d}}+\phi_{0}\right)\right] .
$$

Here $\bar{\tau}_{\mathrm{d}}$ is the acoustic depth below the surface at which the bump is located; $\phi_{0}$ is a constant determined by the phase of the eigenfunctions at the surface of the star; $\left(\delta \Gamma_{1} / \Gamma_{1}\right)_{\bar{\tau}_{\mathrm{d}}}$ is the relative magnitude of the bump in $\Gamma_{1} ; \beta$ measures approximately the half (acoustic) thickness of the bump (see Fig. 1a); and $\tau_{t}$ is the total acoustic radius of the star (viz the sound travel time from centre to surface).

In this study, we fit expression (1) to frequencies of stellar models (Table 1), using a non-linear least-squares method (from [6]). Four parameters are used,: $\bar{\tau}_{\mathrm{d}}, \phi_{0}, \beta$ and $a_{0} \equiv\left(-3 / 4 \tau_{\mathrm{t}}\right)\left(\delta \Gamma_{1} / \Gamma_{1}\right)_{\bar{\tau}_{\mathrm{d}}}$. Only modes with degrees $l \leq 5$ are used in the fit, since we are interested in what might be learned about the distant stars. In order to be able to interpret the results of the fit more easily, we introduce $\delta_{\mathrm{obs}}=-4 \tau_{\mathrm{t}} a_{0} / 3$. This corresponds to the value of $\left(\delta \Gamma_{1} / \Gamma_{1}\right)$ at $\bar{\tau}_{\mathrm{d}}$. The relation of $\delta_{\mathrm{obs}}, \beta$ and $\bar{\tau}_{\mathrm{d}}$ to the helium ionization bump is shown schematically in Fig. 1a. The expected values of $\bar{\tau}_{\mathrm{d}}, \beta$ and $\delta_{\text {obs }}$ are: $\bar{\tau}_{\mathrm{d}} \approx 500-600 \mathrm{~s} ; \beta \approx 100 \mathrm{~s} ;$ and $\delta_{\mathrm{obs}} \approx 0.05$.

The measured values of $\bar{\tau}_{\mathrm{d}}$ are consistent with the expected values. The parameters that are directly relevant to the equation of state and the helium abundance are $a_{0}$ (or equivalently $\delta_{\mathrm{obs}}$ ) and $\beta$. The correlation of the latter with the equation of state is clear for both stellar masses (see Fig. 1b). 
Table 1. ZAMS stellar models of $1 M_{\odot}$ with varying helium abundances $(Y)$, equations of state (EOS), opacities and formulations for convective energy transport. Abbreviations are: SEOS $=$ simple Saha EOS for $\mathrm{H}$ and $\mathrm{He}$ ionization with ad hoc pressure ionization at high pressure; CEFF = the EFF EOS ([5]) with Coulomb correction term (e.g. [2]); SOP = simple power law opacities; CT76 = opacity tables from [4]; MLT = standard mixing length theory; $\mathrm{CM}=$ convection formulation of [1]. Model $Z_{0}$ has SEOS with HeII ionization potential set to zero.

The parameters obtained by fitting equation (1) to model frequencies are also given: the acoustic depth $\bar{\tau}_{\mathrm{d}}$ and half-width $\beta$ are in seconds; $a_{0}$ is in $\mu \mathrm{Hz} ; \phi_{0}$ and $\delta_{\text {obs }}$ are dimensionless.

\begin{tabular}{cccccrcccc}
\hline Mod. & $Y$ & $E O S$ & Opacity & Conv. & $\bar{\tau}_{\mathrm{d}}$ & $\phi_{0}$ & $a_{0} / 2 \pi$ & $\beta$ & $\delta_{\text {obs }}$ \\
\hline$Z_{0}$ & 0.2379 & no HeII & $S O P$ & $M L T$ & & & & & \\
$Z_{1}$ & 0.2378 & $S E O S$ & $S O P$ & $M L T$ & 529 & 3.5 & 1.64 & 108.5 & -0.041 \\
$Z_{2}$ & 0.2378 & $S E O S$ & $S O P$ & $C M$ & 532 & 3.5 & 1.63 & 107.1 & -0.041 \\
$Z_{3}$ & 0.2421 & $C E F F$ & $S O P$ & $M L T$ & 527 & 3.2 & 1.82 & 94.9 & -0.045 \\
$Z_{4}$ & 0.2356 & $C E F F$ & $C T 76$ & $M L T$ & 515 & 3.5 & 1.85 & 95.1 & -0.046 \\
$Z_{5}$ & 0.2356 & $C E F F$ & $C T 76$ & $C M$ & 518 & 3.4 & 1.83 & 93.0 & -0.046 \\
\hline
\end{tabular}
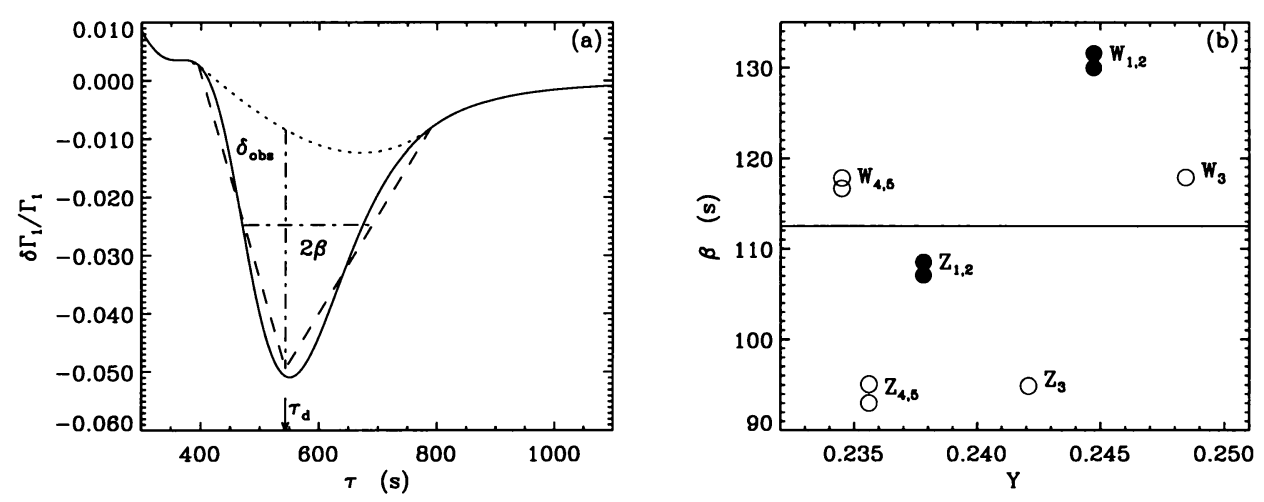

Fig. 1: (a) Schematic of the properties of the ionization zone measurable in the signal $\delta \omega ;$ the half-thickness $\beta$ of the bump, the amplitude $\delta_{\text {obs }}$, and the location $\tau_{\mathrm{d}}$. Model differences $Z_{1}-Z_{0}$ are shown (solid). (b) Inferred values of half-thickness $\beta$ versus helium abundance. Models $W_{1-5}$ have same EOS, opacity and convection treatment as $Z_{1-5}$ but have mass $1.1 M_{\odot}$. Filled circles are for the SEOS; open circles are for the CEFF EOS (see Table 1).

We have shown how the HeII ionization region can be studied using the characteristic signal associated with that layer. The signal can be measured using low degree modes (the ones we hope to observe in other stars). We are gratified that we obtain from the signal values for the parameters with the expected magnitudes (for both $1 M_{\odot}$ and $1.1 M_{\odot}$ stellar models). This shows that the signal is indeed coming from the HeII ionization zone, and that our fitting procedure works.

The width $\beta$ discriminates well the equation of state (see Fig. 1b). Another of our goals has been to measure the helium abundance $Y$. The area of the bump, as determined by $\beta \delta_{\mathrm{obs}}$, is a good measure of $Y$, but further tests are needed to establish how well this can be determined from frequencies.

[1] Canuto V.M., Mazzitelli I. (1991), ApJ 370, 295

[2] Christensen-Dalsgaard J., Däppen W. (1992), A\&AR 4, 267

[3] Christensen-Dalsgaard J., Monteiro M.J.P.F.G., Thompson M.J. (1995), MNRAS 276, 283

[4] Cox A.N., Tabor J.E. (1976), ApJS 342, 1187

[5] Eggleton P.P., Faulkner J., Flannery B.P. (1973), A\&A 23, 325

[6] Monteiro M.J.P.F.G., Christensen-Dalsgaard J., Thompson M.J. (1994), A\&A 283, 247 